\title{
Cautions and Strategy for Treating Chronic Heart Failure by Using Adaptive Servo-Ventilation
}

\author{
Toshiaki Shiomi, MD, PhD; Ryujiro Sasanabe, MD, PhD; \\ Yuko Watanabe; Yutaka Tokunaga, MD
}

$\mathbf{I}$ n 1998, Japanese universal public health insurance approved cover for the home care use of continuous positive airway pressure (CPAP) for sleep apnea syndrome (SAS). The Japanese Ministry of Health, Labor and Welfare criteria for health insurance coverage for CPAP are as follows: "anyone who as the result of polysomnography (PSG) has an apnea hypopnea index (AHI) $\geq 20$ with subjective symptoms such as sleepiness", and "people whose result of the portable monitor test is AHI $\geq 40$, and who have subjective symptoms". Currently, many patients with SAS in Japan receive in-home treatment with CPAP covered by public health insurance. Meanwhile, in 2004, home oxygen therapy (HOT) for patients with chronic heart failure (CHF) and Cheyne-Strokes respiration (CSR) was approved for health insurance coverage with different criteria from CPAP. In 2007, adaptive servoventilation (ASV) was approved as one of home mechanical ventilation by the health insurance, and then ASV has been applied to the treatment of CHF patients whose condition was stable. In order to prescribe ASV, the physician has to have evidence that the patient requires long-term continuous pressure support ventilation that is able to change the tidal volume and respiratory rate. At last, in 2010, the Japanese Circulation Society published "Guidelines for Diagnosis and Treatment of Sleep Disordered Breathing in Cardiovascular Disease; JCS2010 (Chief, Dr Momomura)", and it heated up the discussion about which and how of CPAP, HOT and ASV should be properly prescribed for cardiovascular patients. Accordingly, considering the present status of medical affairs in Japan, we describe cautions and strategy for treating CHF by using ASV.

\section{Article p 661}

Sleep disordered breathing (SDB), including OSA and central sleep apnea (CSA), is a common symptom in patients with heart failure (HF). The prevalence of SDB is reported as 47$76 \%$ of HF patients whose ejection fraction (EF) is reduced, and $55 \%$ of HF patients whose EF remains normal. ${ }^{1,2}$

SDB in CHF is divided into 2 types: OSA and CSA with CSR (CSA-CSR). Some patients have both types. OSA is characterized by pharyngeal airway collapse during sleep, which results in repeated episodes of oxygen desaturation in spite of ongoing respiratory effort and arousals. The distinctive feature of CSR is a respiratory dynamic that leads to CSA without respiratory effort after crescendo-decrescendo changes in tidal volume. It is considered that CSA in CHF patients is caused by increased chemo-responsiveness related to the unstable hemodynamics. ${ }^{3,4}$

The more severe CHF becomes, the more unsatisfactory the prognosis, because severe CHF is apt to be accompanied by CSR. CPAP is an effective device for treating SDB, ${ }^{5}$ thus obviating the factors that can aggravate CHF. Bilevel PAP ${ }^{6}$ and ASV are other types of PAP therapy ${ }^{7-10}$ that are also now used for CHF patients. However, there is not yet enough evidence to prove the long-term efficacy of ASV. For this reason, Takama and Kurabayashi's article in this issue of the Journal, which reports that 1-year use of ASV reduced fatal cardiovascular events and improved the survival rate of HF patients, is considered to be meaningful evidence for encouraging the use of ASV. ${ }^{11}$

Nevertheless, because there is not enough evidence that treating SDB is directly related to reducing the risks for survival and cardiovascular diseases, it is not recommended to routinely assess SDB when evaluating the risks of cardiovascular diseases, and to treat to prevent or manage cardiovascular disease in patients without any symptoms. ${ }^{12}$

Although ASV is expected to be the new approach to treating $\mathrm{CHF}$, because of its efficacy over CPAP, ${ }^{13} \mathrm{ASV}$ requires users to have a strategic management plan. Unfortunately, neither the rate of complications of CSR before the intervention nor the results of the 1-year follow-up evaluation of the improvement on SDB after treatment was described in the authors' previous report. ${ }^{9}$ As Brown's review shows, because most studies to date have lacked titration of undergoing PSG at a sleep laboratory and of follow-up examination to estimate the efficacy of ASV for each individual patient, there is no support for physicians to indiscriminately prescribe ASV for unselected patients to use in their home with the device parameters set to default values. ${ }^{14}$ Consequently, because ASV is a much more complicated device to use than CPAP, it requires greater concern with regard to prescription and management.

Here then are the 3 main points to consider for using ASV as the treatment for CHF in the research and clinical settings. First and foremost, the importance of the diagnosis of SDB (ie, determine if SDB is CSA dominant or OSA dominant), and titration of attended PSG or effect evaluation and concern for compliance. Secondly, it is important to consider side-effects and contraindications such as vomiting, accentuation of airway secretions, potential airway obstruction, active cardiac arrhyth-

The opinions expressed in this article are not necessarily those of the editors or of the Japanese Circulation Society.

Received November 18, 2011; accepted November 20, 2011; released online December 23, 2011

Department of Sleep Medicine, Aichi Medical University School of Medicine, Aichi, Japan

Mailing address: Ryujiro Sasanabe, MD, PhD, Sleep Disorders Center, Aichi Medical School of Medicine, 1-1 Yazakokarimata, Nagakute,

Aichi 480-1195, Japan. E-mail: sasanabe@aichi-med-u.ac.jp

ISSN-1346-9843 doi:10.1253/circj.CJ-11-1331

All rights are reserved to the Japanese Circulation Society. For permissions, please e-mail: cj@j-circ.or.jp 
mias, bullous lung disease, etc. Lastly, although all ASVs look similar, each has a different algorithm depending on the manufacturer. Additionally, there are no comparative data for the effect of each device among its models. ${ }^{15}$ By thorough examination with these considerations, the mechanism of CHF treatment with ASV will be revealed and thus an effective and safe management protocol will be established for this promising device.

\section{References}

1. Chan J, Sanderson J, Chan W, Lai C, Choy D, Ho A, et al. Prevalence of sleep-disordered breathing in diastolic heart failure. Chest 1997; 111: $1488-1493$

2. Sharma B, Owens R, Malhotra A. Sleep in congestive heart failure. Med Clin North Am 2010; 94: 447-464.

3. Bradley TD. Crossing the threshold: Implications for central sleep apnea. Am J Respir Crit Care Med 2002; 165: 1203-1204.

4. Javaheri S. Acetazolamide improves central sleep apnea in heart failure: A double-blind, prospective study. Am J Respir Crit Care Med 2006; 173: $234-237$.

5. Javaheri S, Caref EB, Chen E, Tong KB, Abraham WT. Sleep apnea testing and outcomes in a large cohort of medicare beneficiaries with newly diagnosed heart failure. Am J Respir Crit Care Med 2011; 183: $539-546$.

6. Arzt M, Wensel R, Montalvan S, Schichtl T, Schroll S, Budweiser $\mathrm{S}$, et al. Effects of dynamic bilevel positive airway pressure support on central sleep apnea in men with heart failure. Chest 2008; 134:
$61-66$.

7. Koyama T, Watanabe H, Kobukai Y, Makabe S, Munehisa Y, Iino K, et al. Beneficial effects of adaptive servo ventilation in patients with chronic heart failure. Circ J 2010; 74: 2118-2124.

8. Kasai T, Usui Y, Yoshioka T, Yanagisawa N, Takata Y, Narui K, et al. Effect of flow-triggered adaptive servo-ventilation compared with continuous positive airway pressure in patients with chronic heart failure with coexisting obstructive sleep apnea and Cheyne-Stokes respiration. Circ Heart Fail 2010; 3: 140-148.

9. Takama N, Kurabayashi M. Effectiveness of adaptive servo-ventilation for treating heart failure regardless of the severity of sleep-disordered breathing. Circ J 2011; 75: 1164-1169.

10. Koyama T, Watanabe H, Kobukai Y, Makabe S, Munehisa Y, Iino K, et al. Beneficial effects of adaptive servo ventilation in patients with chronic heart failure. Circ J 2010; 74: 2118-2124.

11. Takama N, Kurabayashi M. Effect of adaptive servo-ventilation on 1-year prognosis in heart failure patients. Circ J 2012; 76: 661-667.

12. Logan AG, Bradley TD. Sleep apnea and cardiovascular disease. Curr Hypertens Rep 2010; 12: 182-188.

13. Philippe C, Stoica-Herman M, Drouot X, Raffestin B, Escourrou P, Hittinger $\mathrm{L}$, et al. Compliance with and effectiveness of adaptive servoventilation versus continuous positive airway pressure in the treatment of Cheyne-Stokes respiration in heart failure over a six month period. Heart 2006; 92: 337-342.

14. Brown LK. Whither adaptive servo-ventilation? Curr Opin Pulm Med 2010; 16: 527-529.

15. Sharma B, McSharry D, Malhotra A. Sleep disordered breathing in patients with heart failure: Pathophysiology and management. Curr Treat Options Cardiovasc Med 2011; 13: 506-516. 